\title{
Etnografia Virtual em Alcoólicos Anônimos em Tempos de Pandemia: Desafios Teóricos e Metodológicos
}

\section{Edemilson Antunes de Campos'}

${ }^{1}$ Escola de Artes, Ciências e Humanidades. Universidade de São Paulo, Brasil | edicampos@usp.br | orcid https://orcid.org/0000-0003-0705-8339

\begin{abstract}
Resumo: O objetivo deste artigo é compreender como os membros de A.A. realizam o tratamento do alcoolismo em meio à pandemia, por meio de uma etnografia virtual em grupos de A.A. no Brasil. Realizou-se uma etnografia virtual em reuniões de recuperação online de grupos de A.A., localizados na Cidade de São Paulo-Brasil e entrevistas, via formulário Google Forms, com membros A.A de diversas cidades brasileiras. Com a pandemia, a etnografia migrou para o espaço virtual, o que tem provocado certos tensionamentos em relação à clássica noção de trabalho de campo em antropologia, ao emprego de técnicas de coleta de dados, tais como a observação participante e as modalidades de entrevistas. Busca-se, assim, contribuir para a compreensão da maneira como os membros de A.A. realizam o tratamento do alcoolismo em reuniões de recuperação online e, por essa via, dos desafios teóricos e metodológicos da etnografia virtual em tempos de pandemia.
\end{abstract}

Palavras-chave: Alcoólicos Anônimos; Covid-19; Etnografia Virtual; Pandemia.

Virtual Ethnography in Anonymous Alcoholics in Times of Pandemic: Theoretical and Methodological Challenges

Abstract: The purpose of this article is to understand how A.A. members carry out the treatment of alcoholism in the midst of the pandemic, through a virtual ethnography in A.A. groups in Brazil. A virtual ethnography was carried out in online recovery meetings of A.A. groups, located in the City of São Paulo-Brazil and interviews, via Google Forms, with A.A members from several Brazilian cities. With the pandemic, ethnography migrated to the virtual space, which has caused certain tensions in relation to the classic notion of fieldwork in anthropology, to the use of data collection techniques, such as participant observation and interview modalities. Thus, it seeks to contribute to the understanding of the way in which A.A. members carry out the treatment of alcoholism in online recovery meetings and, in this way, the theoretical and methodological challenges of virtual ethnography in times of pandemic.

Keywords: Anonymous Alcoholics; Covid-19; Virtual Ethnography; Pandemic.

\section{Introdução}

A pandemia da Covid-19 alterou nosso modo de vida, obrigando a uma reorganização da vida social e econômica, com impacto também na pesquisa acadêmica, que teve que se adaptar a essa nova realidade, com uma revisão de seus métodos e técnicas de pesquisa. Exemplo disso é a pesquisa etnográfica, cujo método se baseia no contato face a face entre pesquisador e pesquisado com o objetivo de se compreender uma determinada realidade sóciocultural. Na pandemia, os pesquisadores foram obrigados a repensar a realização da pesquisa etnográfica, adaptando-a ao contexto virtual.

Todavia, a chamada etnografia virtual nos leva a pensar os desafios teóricos e metodológicos em relação à clássica noção de trabalho de campo em antropologia e as interações entre pesquisadores e pesquisados, bem como o emprego de técnicas de coleta de dados próprias da produção de conhecimento antropológico, como a observação participante e as diversas modalidades de entrevistas e, por fim, as estratégias de análise do material empírico.

Nesse artigo, busca-se compreender como os membros de A.A. realizam o tratamento do alcoolismo em meio à pandemia, por meio de uma etnografia virtual em grupos de A.A. no Brasil. Trata-se de compreender tratamento do alcoolismo em A.A. e os desafios teóricos e metodológicos da etnografia virtual em tempos de pandemia. 


\section{A Etnografia Virtual: Desafios Teóricos e Metodológicos}

O método etnográfico de pesquisa constitui um conjunto de concepções e procedimentos utilizados, tradicionalmente, pela Antropologia, com o objetivo de compreender cientificamente uma dada realidade sociocultural. Desde os estudos clássicos de Malinowiski, (1986), fazer antropologia se confunde com a prática da etnografia no desvelamento de formas de vida e de pensar diferentes das nossas.

A etnografia tem se mostrado fundamental para a compreensão dos aspectos simbólicos da vida social, na medida em que se baseia na ideia de que os comportamentos humanos só podem ser suficientemente compreendidos dentro do contexto sociocultural no qual eles estão inseridos.

Nesse contexto, o trabalho de campo, com a realização de observações in loco sobre os comportamentos vivenciados no território da pesquisa, é fundamental permitindo a construção de um conhecimento baseado no confronto entre nossas hipóteses e nossas observações (VICTORA, KNAUTH \& HANSEN, 2000).

Todavia, com a pandemia muitos pesquisadores tiveram que adaptar suas pesquisas aos espaços virtuais. Nessa linha, muitas pesquisas etnográficas passaram a ser realizada via internet, incorporando-se à chamada etnografia virtual, etnografia on-line ou mesmo netnografia (HINE, 2004).

Dada a especificidade do fazer etnográfico e seu compromisso com o trabalho de campo, essa adaptação para etnografia virtual não é isenta de implicações teóricas e metodológicas. Como bem aponta Hine (2004), um dos principais problemas enfrentados em estudos etnográficos na internet é encontrar uma forma de interação com os sujeitos do estudo adequada ao propósito etnográfico que a pesquisa requer.

Mas, a pesquisa de campo virtual pode ser chamada de etnografia no sentido antropológico? Para Hine (2004), a etnografia virtual problematiza essa ideia tradicional da antropologia de trabalho de campo, na medida em que a pesquisa de campo não implica, necessariamente, mover-se de um lugar para outro e não requer uma observação participante face a face. Em outras palavras, as possibilidades de interações mediadas pela virtualidade nos permitem repensar essa regra da presença física como um fundamento da etnografia virtual, de modo que, para o pesquisador ter a internet como campo de pesquisa tem como propósito fundamental se aproximar da experiência dos sujeitos e explorar as relações sociais estabelecidas nos espaços virtuais.

A etnografia virtual nos leva a refletir sobre os limites do chamado método etnográfico. Como bem aponta Peirano (2014), a etnografia é sempre reinventada e recriada em cada fazer etnográfico. Somos inventores e inovadores da pesquisa etnográfica. Com efeito, a etnografia não se limita a um método de pesquisa, mas sim, a uma inovação teórica, fruto de uma recombinação intelectual a partir dos objetivos da pesquisa.

Ora, a etnografia virtual faz parte dessa inovação teórica fruto tanto das mediações tecnológicas, que cada vez mais fazem parte de nosso dia a dia como das necessidades impostas pela pandemia, que nos leva a recombinar os dados coletados nesse campo virtual à luz dos objetivos da pesquisa. O desafio teórico da etnografia virtual está em elaborar a análise dos dados coletados no campo virtual, que impõe novas exigências ao pesquisador, à luz dos objetivos da pesquisa.

Como observa Miller (2020), o etnógrafo virtual realizará um engajamento online diferente para cada população com que vier a trabalhar. Nessa linha, como existem muitos contextos para pesquisas presenciais, também existe uma diversidade de contextos online e cada um exige uma inovação teórica e metodológica. É o campo que vai informar e formar a pesquisa etnográfica. Se agora o campo é virtual é nele que o pesquisador encontrará as condições de possibilidade para realizar a etnografia, bem como de elaborar a análise teórica a partir dos dados coletados.

Ainda segundo Hine (2004), a inserção do pesquisador no campo virtual pode se dar de duas formas, a depender das características do campo: "lurker" ou "insider". 
A primeira, diz respeito a uma observação participante mais "silenciosa", sem interferência ou interação com o meio investigado. Dito de outra forma, nesse tipo de observação o pesquisador observa, mas não intervém no campo empírico. A segunda é mais relacional, "ativa", na qual há uma proximidade e interação significativa entre o pesquisador e os sujeitos da pesquisa (HINE, 2004; PEREIRA NETO et al., 2015).

A etnografia virtual já é feita por pesquisadores brasileiros que estudam as relações socioculturais na internet. Exemplo disso é o trabalho de Pereira Neto e colaboradores (2015) realizaram uma etnografia virtual em três grupos on-line no Facebook que reúnem pessoas que vivem com diabetes, com hepatite $\mathrm{C}$ e com HIV/aids, onde analisaram o empoderamento proporcionado pela internet e a validade dos saberes médicos na visão desses usuários diante das suas condições. Ou ainda, o trabalho de Separavich e Canesqui (2012), que analisaram as representações e os relatos da experiência com a menopausa contidos no site brasileiro da internet "Menopausa - o site da mulher madura". Para os autores, tratava-se de um espaço de interatividade, no qual procuraram identificar aspectos do climatério/menopausa, da vivência desse processo e das ações de cuidados adotadas por mulheres que passavam por essa fase da vida. E o trabalho de Melo (2020), no qual estuda grupos de internet de pessoas que vivem com o HIV/aids (PVHA), apontando o modo como a interação em espaços virtuais é fundamental para a superação dos preconceitos e para a construção identitária dos membros do grupo virtual.

Esses estudos evidenciam que a etnografia virtual pode e deve ser conduzida pelo pesquisador de maneira ética e como uma oportunidade de compreensão dessa nova maneira de se relacionar e de se vivenciar as experiências nesse contexto de isolamento social imposto pela pandemia.

\section{A Etnografia Virtual em Grupos de Alcoólicos Anônimos em Tempos de Pandemia}

Para dar conta dos objetivos propostos foi realizada uma etnografia virtual, com observação participante, em reuniões de recuperação on line de grupos de A.A., localizados na Cidade de São Paulo - Brasil e também entrevistas, por meio de formulário Google Forms, com membros de A.A. de diversas cidades brasileiras. Essa pesquisa foi aprovada pelo Comitê de Ética em Pesquisa, da Escola de Artes, Ciências e Humanidades, da Universidade de São Paulo (CEP-EACH/USP).

O A.A. é, de acordo com sua literatura oficial, "uma irmandade de homens e mulheres que compartilham suas experiências, forças e esperanças, a fim de resolver seu problema comum e ajudar outros a se recuperarem do alcoolismo" (ALCOÓLICOS ANÔNIMOS, 1996).

Com a pandemia, muitos grupos de A.A. suspenderam as reuniões de recuperação presenciais, bem como demais atividades que pudessem causar aglomeração de pessoas e, consequentemente, a contaminação pelo Coronavírus. Dessa forma, as reuniões passaram a ser virtuais, de modo que se utilizando da internet, os membros de A.A. pudessem dar continuidade ao tratamento do alcoolismo.

A utilização das mídias sociais não é uma novidade para o A.A., que usa da internet para divulgar suas informações e também para promover troca de experiências entre seus membros, tanto por meio da página na internet da Junta de Serviços Gerais de Alcoólicos Anônimos no Brasil (JUNAAB) ${ }^{1}$, como de um grupo privado no Facebook, cujo nome é: Alcoólicos Anônimos Brasil (AA). O que passou a ser novo com a pandemia é centralidade que as reuniões virtuais passaram a ocupar no tratamento do alcoolismo. Com a impossibilidade de se fazer reuniões presenciais, alguns grupos de A.A. passaram a promover reuniões on-line, o que exigiu uma adaptação de seus membros a essa nova realidade imposta pela pandemia.

${ }^{1}$ Cf. https://www.aa.org.br/ 
Se a pandemia exigiu essa reorganização de A.A., o mesmo aconteceu na pesquisa etnográfica, que também teve que ser feita na forma virtual. Na página da internet da JUNAAB é possível saber quais grupos de A.A., no Brasil, realizam reuniões à distância ${ }^{2}$. A Cidade de São Paulo conta com 121 grupos de A.A, mas, apenas 19 grupos promovem reuniões de recuperação on-line abertas, com média de uma hora e meia de duração e cujo acesso se dá tanto pela plataforma Google Meet, como pelo Zoom³. Há também reuniões virtuais fechadas somente para membros de A. $A^{4}$.

Na página de A.A. também se encontra orientações sobre o modo como deve transcorrer o acesso e a dinâmica das reuniões. Com efeito, é proibida a gravação das reuniões e todos devem manter a câmera de vídeo desligada em respeito ao princípio do anonimato ${ }^{5}$. Há também ressalvas quanto aos cuidados para se evitar interrupções das reuniões, em relação às movimentações na casa dos participantes e quanto à vestimenta.

Mas, como são as reuniões de recuperação virtuais? Quais as diferenças entre as reuniões virtuais e presenciais? Foi munido dessas questões que eu entrei na página da internet de A.A. e vi os dias e horários das reuniões de recuperação virtuais e iniciei a pesquisa de campo, em dois grupos virtuais de A.A., aqui denominados Grupo 1 e Grupo 2, com reuniões todos os dias da semana das $19 \mathrm{~h} 00$ às $20 \mathrm{~h} 30$, pela plataforma Google Meet e Zoom.

No início da reunião, o coordenador lê um preâmbulo, com a reflexão diária, extraída dos escritos de Bill Wilson (ALCOÓLICOS ANÔNIMOS, 2000), fundador do A.A., e chama a todos para fazerem a Oração da Serenidade ${ }^{6}$. O coordenador chama a atenção dos membros sobre os cuidados que devem ser tomados em relação ao anonimato. Após, dá-se início as partilhas com cada participante tem 10 minutos para compartilhar suas experiências tanto do tempo do alcoolismo ativo, como do tempo de recuperação em A.A.. A partir daí, o coordenador convida os membros de A.A. a fazer a partilha, que é ouvida por todos em silêncio: "Meu nome é Roberto, sou um doente alcoólico em recuperação e participo da reunião para não ter consciência do meu alcoolismo, que preciso de ajuda e agradeço ao Poder Superior e aos Alcoólicos Anônimos por eu não voltar a beber. Eu tenho um problema com minha ex-mulher, mas sei que pelo meu alcoolismo, eu não posso perder a cabeça". (Roberto, reunião de recuperação virtual, Grupo 1)). "Meu nome é Cris e sou doente alcoólico em recuperação. Participo das reuniões virtuais, mas sinto falta do grupo, tenho saudade, eu preciso sentir a unidade presencialmente". (Cris, reunião de recuperação virtual, Grupo 2)). "Meu nome é Fátima, uma alcoólica em recuperação. Hoje, eu estou triste porque não tenho filhos, mas eu cuidei de um sobrinho que me considera como sua mãe, mas, a namorada de meu irmão não cuida dele". (Fátima, reunião de recuperação virtual, Grupo 1). "Meu nome é Elisa, sou alcoólatra em recuperação e agradeço ao Poder Superior por eu não voltar a beber. Sou filha de pai alcoólatra e comecei a beber em casa. Depois comecei a beber socialmente, e minhas amigas me diziam de meu comportamento quando eu bebia; que eu perdia o controle, muito vexatório. Eu ofendia meus amigos, minha mãe, até que eu resolvi procurar o A.A.." (Elisa, reunião de recuperação virtual, Grupo 2). Ao longo da reunião, o coordenador lê mensagens de incentivo para que os membros continuem sua recuperação. Após uma hora de reunião, o coordenador lê a sétima tradição, que diz sobre as contribuições financeiras voluntárias para manter o funcionamento do grupo e também passa informações sobre as atividades promovidas pelo grupo.

${ }^{2}$ Cf. https://sites.google.com/aa.org.br/g-suite/reunioes-a-distancia/lista-das-reunioes

${ }^{3}$ Cf. https://sites.google.com/aa.org.br/g-suite/reunioes-a-distancia/lista-das-reunioes/saopaulo\#h.njhz8cxlq6j3

${ }^{4}$ Cf. https://sites.google.com/aa.org.br/g-suite/reunioes-a-distancia/reunioes-para-membros

${ }^{5}$ A Décima Segunda tradição de A.A. aponta: "O anonimato é o alicerce espiritual de todas as nossas tradições, lembrando-nos sempre da necessidade de colocar os princípios acima das personalidades".

6 A Oração da Serenidade é feita no início e no final das reuniões de recuperação, virtuais e presenciais: "Concedei-nos Senhor, a serenidade necessária para aceitas as coisas que não podemos modificar. Coragem, para modificar aquelas que podemos. E, Sabedoria, para discernir uma das outras". 
Ao final, da reunião o coordenador faz o encerramento da reunião, convidada a todos a fazer novamente a Oração da Serenidade. Todos se despedem, com votos de boa noite e o link da reunião virtual é encerrado.

Além da observação participante em reuniões de recuperação virtuais, também foram realizadas entrevistas, via formulário Google Forms, com 35 membros de A.A. de diferentes cidades brasileiras. O formulário possuía um registro de consentimento, no qual se explicava os objetivos da pesquisa, a garantia do anonimato e o direito de não participar da pesquisa e o consentimento para divulgação dos dados obtidos na entrevista. Além disso, o formulário possuía o registro de dados pessoais, tais como: nome, idade, estado civil, naturalidade, escolaridade, profissão, tempo de A.A. e grupo base. Os participantes eram convidados a responder a seguinte pergunta: "A pandemia afetou o tratamento do alcoolismo em A.A.? Se sim, como?".

Dentre os entrevistados, tem-se que 19 são do Estado de São Paulo - Capital, 10 são do Estado de São Paulo, de cidade do interior e litoral, 04 são do Estado da Bahia, 01 é do Estado do Rio de Janeiro e 01 do Distrito Federal.

As entrevistas, através do Google forms, foram fundamentais para a pesquisa, pois permitiram uma aproximação mais intensa com os membros de A.A. em tempos de pandemia. Com efeito, se, nas reuniões virtuais, foi possível observar a maneira como ocorre a recuperação, nas entrevistas é possível estabelecer um maior controle sobre os dados, de maneira a compreender a maneira como os membros de A.A. avaliam os efeitos da pandemia para o tratamento do alcoolismo.

\section{Alcoólicos Anônimos e a Pandemia: os Desafios da Etnografia Virtual}

A análise dos dados visa compreender tanto os efeitos da pandemia no tratamento do alcoolismo em A.A. como os desafios da etnografia virtual, particularmente, em relação à entrada no campo de pesquisa, à interação entre pesquisador e pesquisado, à utilização das técnicas de pesquisa, observação participante e à análise dos dados obtidos.

Todos os entrevistados sinalizaram que a pandemia afetou o tratamento do alcoolismo em A.A., obrigando os grupos a se adaptarem a essa nova situação. É o que vemos, por exemplo, na resposta a seguir: "Quando as primeiras quarentenas foram decretadas, no mundo inteiro, aliás, acredito que todos nós sofremos um impacto violento (...) Mas logo começamos a "abrir picada" começando pela boa e velha terapia do telefone, do Whatsapp e das videoconferências. Inúmeros grupos passaram a abrir reuniões em meio virtual e logo a JUNAAB passou a disponibilizar esse canal para nossas áreas (conjuntos de distritos) e distritos (conjuntos de grupos), de modo que, de março de 2020 até agora, centenas delas passaram a ser oferecidas." (Elaine, 60 anos, casada, São Paulo-Capital, 28 anos de A.A.). Os membros de A.A. entrevistados também reconhecem que tiveram que se adaptar aos novos tempos para dar continuidade à recuperação: "A frequência às reuniões é a base da recuperação principalmente para membros novos/as. Mas, entendo que a grande sacada do AA, a chave de ouro, é o programa dos 12 passos. Adaptar-se as mudanças. Para os membros/as mais conservadores/as foi e creio que ainda está sendo um pouco traumático." (Max, 43 anos, solteiro, Rio de Janeiro, 5 anos de A.A.). Ou ainda, "a pandemia ajudou, pois as plataformas nos favoreceram no acesso a inúmeras reuniões de literatura e temática, que aprendemos muito e estamos em constante aprendizado" (Luiza, 48 anos, casada, Distrito federal, um ano e 06 meses de A.A.).

Mas, a pandemia também criou dificuldades de acesso, pois, nem todos conseguem participar das reuniões virtuais: "a falta de reuniões presencias é um problema real. Eu tenho a condição de ter internet e bom celular computador e estar fazendo minha recuperação online. Como nas escolas, as reuniões à distância não atingem a todos" (Lidiane, 61 anos, divorciada, Bahia, 19 anos de A.A.). 
Um aspecto importante ressaltado nas entrevistas foi a possibilidade de atrair novos membros, ou de "levar a mensagem de A.A." para aqueles que precisam de ajuda: "as reuniões em meio virtual começaram a se tornar "portas de entrada" capazes de atrair jovens e mulheres como nunca antes na história de A.A. Como também pessoas que vivem em regiões onde não existem grupos locais de A.A.." (Elaine, 60 anos, casada, São Paulo-Capital, 28 anos de A.A.).

Se as entrevistas revelaram as dificuldades enfrentadas pelos membros de A.A. para realizarem o tratamento do alcoolismo em tempos de pandemia, a observação participante nas reuniões de recuperação virtuais mostrou o modo como a troca de experiências, mesmo online, é fundamental para a consolidação tanto da noção do alcoolismo concebido como "doença fatal" como da identidade do "doente alcoólico". Em estudo anterior (CAMPOS, 2010), mostramos como o A.A. opera como um modelo terapêutico no qual se constrói os significados da experiência do alcoolismo como doença e de seus membros como doentes alcoólicos em recuperação. Ao longo das partilhas, os membros de A.A. vão tecendo suas experiências vividas, na vida familiar e no trabalho, entrelaçando-as com a doença do alcoolismo. Com efeito, a etnografia virtual, com a observação das reuniões online, permite compreender o modelo terapêutico de A.A. em ato, ou seja, no momento em que seus membros se reúnem virtualmente e compartilham suas experiências para se recuperarem do alcoolismo e se reconhecerem como doentes nessa pandemia.

A análise dos dados coletados na etnografia virtual aponta que para a reafirmação da condição de doente alcoólico, que necessita das reuniões virtuais como um ambiente que permite as trocas de experiências e, por essa via, o tratamento do alcoolismo, concebido como uma doença fatal, em tempos de pandemia.

A pandemia afetou o método de pesquisa e as técnicas para coleta de dados, impondo desafios ao pesquisador. Com efeito, um desafio fundamental é a entrada no campo de pesquisa, que exige uma preparação prévia, com contato com o coordenador das reuniões ou com membros de A.A. ativos. Esse contato prévio é importante para a entrada no campo, o que não diminuiu a curiosidade e o estranhamento provocado pela presença de um pesquisador "não alcoólico" na reunião. Em estudo anterior, apontamos a importância de se responder às questões feitas pelos membros de A.A. e de se deixar claro os objetivos da pesquisa (CAMPOS, 2017).

Um aspecto fundamental da etnografia virtual diz respeito ao anonimato, que, como vimos, é um princípio fundamental das Tradições de A.A.. Assim, nas reuniões online o anonimato virtual, proporcionado pela internet, na qual o participante da reunião pode não abrir a câmera, ou mesmo, ter um pseudônimo como identificação, garante uma espécie de "proteção", uma vez que o risco de preconceito, discriminação e rejeição é reduzido por ter a identidade protegida pelo anonimato.

Uma vez na sala de reunião virtual de A.A., o pesquisador deve manter uma discrição e fazer a observação participante, com anotações detalhadas em diário de campo de toda a dinâmica da reunião. Embora se exija a discrição, o pesquisador pode reproduzir certas práticas realizadas durante as reuniões (FAINZANG, 2002), como, por exemplo, fazer a oração da serenidade, ao início e término das reuniões. Desse modo, o pesquisador coloca em prática certos códigos ritualizados, que assinalam seu envolvimento com o grupo. Com isso, sua posição dentro do grupo virtual vai se consolidando cada vez mais, de maneira a facilitar o acesso às informações necessárias para o trabalho de campo, delimitando seu "lugar" entre os membros de A.A..

É fundamental que o pesquisador participe com regularidade das reuniões, para que possa colher as informações essenciais de acordo com os objetivos da pesquisa. Durante a reunião virtual, é importante observar o modo como ocorrem as partilhas, a entonação da voz, as emoções, os sentimentos, são elementos extremamente significativos para a etnografia. É importante também observar se algum dos participantes da reunião abre a câmera, de modo a revelar sua identidade, mesmo com a recomendação para não fazê-lo. 
Uma atenção também é importante para os silêncios, sobretudo, daqueles que não se sentem a vontade de fazerem a partilha no meio virtual, anotando-se no diário de campo, quantos membros fizeram a partilha e quantos não quiseram fazê-la.

Em se tratando dos riscos decorrentes da pesquisa para os entrevistados, algumas estratégias de enfrentamento devem ser utilizadas pelo pesquisador, como por exemplo, diante do constrangimento no momento de responder a alguma pergunta do roteiro de entrevista, informar ao entrevistado que ele terá a possibilidade de se retirar da pesquisa em qualquer fase da coleta de dados sem nenhum prejuízo.

Com o objetivo de garantir a consideração de todos os interesses envolvidos no trabalho e coleta de dados, cada entrevistado deve tomar conhecimento do Registro de Consentimento, tal como previsto na Resolução 510, Conselho Nacional de Saúde (Brasil, 2016), no qual autorizará sua participação, bem como a utilização dos dados obtidos em resposta em futuras publicações.

\section{Conclusão}

Este artigo buscou compreender a maneira como os membros A.A. se adaptaram a essa nova realidade imposta pela pandemia para realizarem o tratamento do alcoolismo, bem como os desafios da etnografia virtual.

Com efeito, foi realizada observação participante em reuniões virtuais de A.A., nas quais os membros do grupo podem compartilhar suas experiências dos tempos do alcoolismo ativo e também da pandemia, reafirmando sua condição de doentes alcoólicos em recuperação. Já as entrevistas com membros de A.A. apontaram os efeitos da pandemia para o tratamento do alcoolismo, nas quais, se reconhece as dificuldades para o acesso à reunião virtual, a falta da reunião presencial e do contato direto entre os membros do grupo. Por outro lado, os entrevistados também ressaltam as possibilidades abertas pelas reuniões virtuais, tornando possível atrair novos membros e aumentar o acesso à doutrina de A.A..

Nessa linha, a etnografia permite ao pesquisador de investigação qualitativa um contato direto com o universo da pesquisa de campo, dentro do contexto onde os sujeitos estão inseridos. A entrevista e a etnografia virtual são instrumentos fundamentais para a compreensão da relação entre o dizer e o fazer, isto é, entre aquilo que os membros de A.A. dizem sobre os efeitos da pandemia em sua recuperação e aquilo que eles fazem nas reuniões virtuais para tratarem do alcoolismo.

A etnografia virtual deve ser precedida de uma preparação prévia para entrada no campo de pesquisa, de modo a permitir a coleta de dados para posterior análise. Para isso, o pesquisador deve ter presente os objetivos e os fundamentos teóricos da pesquisa que irão nortear a realização do trabalho do campo.

Com efeito, o ambiente virtual se mostra tanto o contexto possível para a realização da pesquisa etnográfica, exigindo do pesquisador um compromisso ético para a realização do trabalho de campo, como permite aos membros de A.A. a continuidade do tratamento do alcoolismo, por meio das reuniões de recuperação online, nesses tempos de pandemia.

\section{Agradecimentos}

Agradeço à FAPESP pelo apoio nessa pesquisa, processo número: 2017/18535-9. Agradeço também às alunas: Andressa Gabrielle Teixeira do Valle, Mariana Melo e Marselle Ferreira Salles, que ajudaram na coleta das entrevistas. 


\section{Referências}

Alcoólicos Anônimos (1996). O Grupo de AA: onde tudo começa. São Paulo: JUNAAB - Junta de Serviços Gerais de Alcoólicos Anônimos.

Alcoólicos Anônimos (2000). Reflexões diárias, São Paulo: JUNAAB - Junta de Serviços Gerais de Alcoólicos Anônimos.

BRASIL. Resolução ํㅜ 510, de 07 de abril de 2016. Dispõe sobre as normas aplicáveis a pesquisas em Ciências Humanas e Sociais. Diário Oficial [da] República Federativa do Brasil, Brasília, DF, 24 maio 2016. Disponível em:

https://bvsms.saude.gov.br/bvs/saudelegis/cns/2016/res0510_07_04_2016.html (Acesso em 18.04.2021).

Campos. E.A. (2010). "Nosso remédio é a palavra”: uma etnografia sobre o modelo terapêutico de Alcoólicos Anônimos. Rio de Janeiro: Editora FIOCRUZ.

Campos. E.A. (2017). Etnografia na pesquisa em saúde: alcoolismo, doença e significado em um grupo de Alcoólicos Anônimos. Investigação Qualitativa em Ciências Sociais, CIAIQ 2017, 3: 537-545.

Fainzang, S. (2002). De l'autre côté du miroir: réflexions méthodologiques et épistémologiques sur l'ethnologie des anciens alcooliques. In. De l'ethnographie à l'anthropologie réflexive: nouveaux terrains, nouvelles pratiques, nouveaux enjeux. Ghasarian, C. (org.). Paris: Armand Colin, 63-71.

Hine, C. Etnografía virtual. Barcelona: Editorial UOC, 2004.

Malinowiski, B. (1986). Introdução: o assunto, o método e o objetivo desta investigação. In. Coleção Grandes Cientistas Sociais. São Paulo: Àtica, 24-48.

Melo, L.P. (2020). Sofrimento social e ajuda mútua entre pessoas que vivem com HIV/aids em uma rede social na internet. Relatório de Pesquisa. São Paulo: FAPESP.

Miller, D. Como conduzir uma etnografia durante o isolamento social. Blog do Sociofilo, 2020. Disponível em: https://blogdolabemus.com/2020/05/23/notas-sobre-a-pandemia-comoconduzir-uma-etnografia-durante-o-isolamento-social-por-daniel-miller. (Acesso em 18.04.2021).

Peirano, M. (2014). Etnografia não é método. Horizontes Antropológicos, 20(42), 377-391

Pereira neto, A.P. et al. (2015). O paciente informado e os saberes médicos: um estudo de etnografia virtual em comunidades de doentes no Facebook. História, Ciências, SaúdeManguinhos, Rio de Janeiro, 22: 1653-1671, 2015.

Separavich, M.A.; Canesqui, A.M. Análise das narrativas sobre a menopausa de um site brasileiro da internet. Interface: Comunicação, Saúde, Educação, Botucatu, 16(42): 609-622, 2012.

Victora, C., Knauth, D. \& Hansen, M. (2000). Pesquisa qualitativa em Saúde: uma introdução ao tema. Porto Alegre: Tomo Editorial. 